\title{
Algunas reflexiones sobre ética y estudio de los varones en la investigación demográfica: una experiencia personal en la IUSSP
}

\section{Juan Guillermo Figueroa Perea*}

Asistir a la XXV Conferencia Internacional de Población fue una experiencia enriquecedora. Organizada por la Unión Internacional para el Estudio Científico de la Población, y celebrada en Tours, Francia, la Conferencia contó con 180 mesas redondas y más de 600 carteles académicos, que permitieron conocer temas emergentes, temas estudiados pero que ahora cuentan con nuevas aproximaciones, y temas que continúan siendo investigados, pero que al contar con información actualizada permiten realizar comparaciones en el tiempo. A la vez, la Conferencia también nos ilustró sobre la variedad de los recursos disponibles en los diferentes países para llevar a cabo los procesos de investigación, como son las diversas metodologías, las problemáticas de estudio, y los recursos humanos y materiales. Una reunión así permite también identificar la manera en que la demografía se ha vinculado con otras disciplinas, diversificando su objeto de estudio, lo cual es positivo en la medida en que permite enriquecer sus criterios de interpretación, aunque para algunos autores puede convertirse en una limitante si se impide el análisis y el seguimiento de los aspectos fundantes de la disciplina, los cuales han ido moldeando la historia de la misma.

Como sucede en tantos congresos académicos que recurren a sesiones simultáneas (táctica necesaria para poder presentar 900 ponencias en cinco días de trabajo, exponer 150 carteles diariamente y propiciar el diálogo entre los diferentes autores), muchas personas optamos por asistir a las sesiones más cercanas a los temas que investigamos, así como a las que presentaban temas novedosos, paradigmáticos o hasta los considerados "exóticos o extravagantes", a la luz del contexto en el que se vive (aunque tengan una pertinencia evidente en los lugares en donde se desarrollan las investigaciones que los abordan).

Con estos considerandos como referencia, las siguientes notas pretenden recuperar algunos de los elementos relevantes de las mesas

* Profesor investigador del Centro de Estudios Demográficos, Urbanos y Ambientales de El Colegio de México. Correo electrónico: jfigue@colmex.mx.

ESTUDIOS DEMOGRÁFICOS Y URBANOS, VOL. 21, NÚM. 1 (61), PP. 227-237 
redondas y carteles que tuve oportunidad de atender, y de algunos trabajos que sólo pude conocer mediante los resúmenes del libro y del disco compacto del congreso (excelentes insumos a ser consultados por las personas interesadas en el tema de la población).

Un importante tema que apareció de múltiples formas en la Conferencia es el relativo a la dimensión ética subyacente a los procesos de generación de información sobre la población, al uso de dicha información en la medida en que repercute en las políticas públicas y en otras acciones sociales, pero también a las omisiones sobre lo no investigado, y a las percepciones, opiniones y valoraciones que tienen quienes investigan y hacen política pública alrededor de las decisiones que toma la población. Por ejemplo, un trabajo presentado por el director de un instituto de estudios demográficos en Francia cuestionaba si existe una ética específica para la demografía, ${ }^{1}$ y para ello mostraba las características diferenciales que tiene el proceso de obtención de información en los análisis micro y macrodemográfico. Ese autor exponía que los vínculos y la interacción con los sujetos de investigación son diferentes, como lo es también el tipo de disciplinas que apoyan dichas investigaciones; en ambos casos el proceso que busca generar conocimientos posee dilemas específicos, que tienen mucho que ver con la interpretación vigente sobre el objetivo del quehacer demográfico y los compromisos de quienes lo llevan a la práctica.

En esta vertiente son interesantes las reflexiones que se hicieron en la mesa sobre historia y epistemología de la demografía y de la sociología (discutiéndolas de manera conjunta). Se problematizaba hacia dónde se dirige la disciplina una vez que se ha enriquecido y diversificado la visión de sus objetos de estudio mediante el intercambio con otras áreas del conocimiento; ${ }^{2}$ también se cuestionaba el papel que ha ido adquiriendo la metodología como una prioridad en ejercicios de investigación, así como la invisibilidad de la teoría en dichos trabajos. Ello llevó a un ponente a cuestionar si no existía una especie de "terrorismo estadístico", y a otro a reflexionar sobre las frustraciones asociadas al proceso de entrenar a la gente en las metodologías cualitativas en un contexto en que pareciera que el ambiente político y cultural es poco receptivo a las mismas. ${ }^{3}$ Dicha reflexión es cercana a

${ }^{1}$ Francois Héran, "Is there a specific ethics for demography?".

${ }^{2}$ Dominique Tabutin, "Vers quelle(s) démographie(s)? Atouts, faiblesses et évolutions depuis 50 ans"; Yves Charbit, "Epistemology of population doctrines and theories".

${ }^{3}$ Hania Sholkamy, "The frustrations and future of teaching qualitative methods to researchers in the Arab World". 
un tipo de reconstrucción histórica de la demografía próxima al ambiente ideológico de diferentes momentos, a coyunturas políticas y demandas internacionales, sin que ello signifique negar sus estrechos lazos con los avances teóricos asociados al estudio de la población. ${ }^{4}$

Como muestra de los vínculos que tiene el uso de la información demográfica con cuestiones políticas e ideológicas, y como ejemplo de la necesidad de llegar a ciertos acuerdos de tipo ético, se presentó un trabajo en la sesión sobre ética y derechos humanos que provocó controversia debido a que evidenciaba que la oficina del Censo de Población de Estados Unidos, al disponer de información desagregada por barrios o por zonas geográficas muy bien delimitadas, puede hacer visibles a personas que políticamente podrían llegar a ser rechazadas, agredidas y discriminadas en el contexto actual de dicha nación y en el internacional. ${ }^{5}$ La autora mostraba que en este caso las leyes para hacer más transparente la difusión de la información podrían generar resultados adversos y violentos contra grupos específicos; reflexionaba también sobre esa problemática situación desde el punto de vista ético, ya que formalmente no podía ser calificada como ilegal pues no existe ninguna ley que la prohíba. ${ }^{6}$ La investigadora además alertaba sobre las consecuencias de tipo metodológico y de confiabilidad en la información, ya que esta práctica puede llegar a desprestigiar el trabajo de la oficina de censos, reducir el nivel de confianza que se puede tener en la misma, y a la larga generar datos más imprecisos sobre los fenómenos de estudio, precisamente porque las personas deliberadamente quieran ocultar información una vez que ésta ha llegado a ser utilizada en su contra, a pesar de la promesa de confidencialidad.

En la vertiente del rigor académico, otra modalidad de la problemática ética que permeó algunos de los trabajos de la conferencia la constituye un trabajo presentado por un demógrafo peruano a propósito de lo que el distinguió como inducción, deducción, analogía y abducción, en tanto recursos para generar conocimiento con sus especificidades lógicas y epistemológicas, contando cada uno con procedimientos para legitimar un avance en el saber en la demografía. ${ }^{7}$

\footnotetext{
${ }^{4}$ Melinda Mills, "Theory and 'demography': a longitudinal empirical analysis from 1964 to 2004"; John Hobcraft, "Population paradigms: pathways and processes".

${ }^{5}$ Samia El-Badry y David A. Swanson, "Controversy over providing special census tabulations to government security agencies in the United States: the case of Arab-Americans".

${ }^{6}$ José Eustaquio Diniz Alvez, "Population, citizenship and human rights in Brazil: elements for a system of indicators".

${ }^{7}$ José Octavio Escobedo Rivera, "Paradigmas epistemológicos e inferencias lógicas en la investigación demográfica”.
} 
Una modalidad más del debate ético emergió en las sesiones denominadas "plenaria-debate", en las cuales se hacía una pregunta sobre una problemática ético demográfica (por llamarla de alguna manera) que podía ser contestada con un sí o con un no, y obviamente con muchos matices. ${ }^{8}$ Sin necesidad de llegar a un consenso, se invitaba a dos personas a que contestaran afirmativamente y a dos de manera negativa; se les pidió también que elaboraran una reflexión para respaldar su respuesta. A partir de ello se invitaba a los asistentes a que hicieran lo mismo, aunque en un tiempo más reducido; es decir que compartieran otras argumentaciones para respaldar una postura positiva o negativa ante la pregunta formulada inicialmente. La idea era poner sobre la mesa los argumentos y de alguna manera estimular que todos los asistentes escucharan posturas que podrían respaldar sus puntos de vista, cuestionarlos o llevarlos a matizar sus respuestas sobre asuntos tan complejos como si debe existir una preocupación sobre el futuro de la población europea dado su proceso de envejecimiento y la disminución de su fecundidad, o si en dicho contexto tendrán éxito las políticas dirigidas a incrementar nuevamente el nivel de la descendencia, y finalmente si se tiene o no el derecho a decidir el sexo de los hijos, tomando en cuenta que se ha peleado tanto por reconocer el derecho de las personas a decidir sobre su reproducción.

Si bien un conocimiento más riguroso de los temas podría llevar a que mucha gente optara por una respuesta matizada o condicionada, un formato de debate como éste, frecuente en algunas reuniones de tipo filosófico, es una novedad interesante en el ámbito de los estudios de población, en particular cuando la gente está dispuesta a escuchar antes de tomar una postura defensiva de sus respectivos puntos de vista. Este tipo de ejercicios muestra cómo la demografía no está exenta de una reflexión ética mucho más sistemática, la cual es de una gran utilidad al hacer evidente que la ética va mucho más allá de la mera formulación de la bondad o la maldad de ciertos comportamientos de las personas, para explorar los supuestos de los juicios morales, de las normas sociales y de las valoraciones individuales que se tienen en diferentes sociedades en un mismo momento histórico y en una misma sociedad a lo largo del tiempo. Es decir, diferentes debates mostraron las aristas ético políticas y metodológicas asociadas a diversos temas de

${ }^{8}$ Plenarias: a) "Visión de la Conferencia Internacional sobre Población y Desarrollo: ¿Cuán lejos hemos llegado en estos once años de travesía?", $b$ ) "Will policies be effective to raise Fertility in Low-Fertility Countries?", c) "Should couples have the right to choose the sex of their children?". 
la investigación demográfica. Si bien la parte metodológica y la política han sido más documentadas en la literatura demográfica, es más limitado lo que se ha hecho en la parte ética.

Dicha situación se debe en parte a que se ha asociado que los cuidados éticos están más cercanos al trabajo clínico, biomédico o de experimentación con seres humanos. Como consecuencia se vuelve invisible el hecho de que la demografía, junto con otras disciplinas, ha generado conocimientos que han alimentado la definición de las políticas que inciden sobre los derechos de las personas y sobre la construcción de las identidades de género, ${ }^{9}$ pero que también repercuten sobre acciones que pueden ser clasistas (como controlar el crecimiento de la población en ciertas regiones del mundo, mientras que en países del norte se facilita el acceso a técnicas de reproducción asistida), generando en cada contexto diferentes tipos de dilemas, los cuales podrían ser acompañados de paradigmas de reflexión ética paralelos al trabajo de investigación sobre población y a la definición de políticas públicas en dicho ámbito. ${ }^{10}$

Una segunda vertiente a destacar dentro de los temas abordados en la XXV Conferencia Internacional sobre Población es la relacionada con la referencia a los varones, en tanto población masculina, dentro de los diferentes momentos de los espacios reproductivos, ya que poco a poco este tema ha ido adquiriendo visibilidad, en particular después de la sugerencia política de la conferencia de El Cairo en 1994 alrededor del tema de la salud reproductiva, de la reflexión más académica de la IUSSP en 1995 en el seminario sobre fecundidad y ciclos de vida masculina celebrado en Zacatecas, y del seminario sobre formación familiar y comportamiento reproductivo de los varones, que tuvo lugar en Buenos Aires en 1998.

En esta conferencia de Francia de 2005 el abordaje de la población masculina y los espacios reproductivos tuvo diferentes matices, incluyendo el análisis de la fecundidad de dicha población ${ }^{11}$ (más

${ }^{9}$ Carole Brugeilles, "Politique démographique au Mexique: 1'expérience de trois génerations de femmes".

${ }^{10}$ Henry P. David y Zdenek Matejcek, "Born unwanted: 35 years later"; Dudley L. Poston Jr. y Karen Glover, "Too many males: marriage market implications of gender imbalances in China"; Leela Visaria, "Sex selective abortion in India: some empirical evidence"; Shuzhuo Li, Yan Wei y Marcus W. Feldman, "Son preference and induced abortion in rural China: findings from the 2001 National Reproductive Health Survey"; Wei Chen, "Sex-selective abortions: evidence from rural East China"; Quanbao Jiang, Marcus W. Feldman y Xiaoyi Jin, "Estimation of the number of 'missing girls' in China: 1900-2000".

${ }^{11}$ Lisbeth B. Knudsen y Anne-Marie Nybo Andersen, "Trends in delayed onset of fertility among men". 
desde la lógica de los registros vitales que de las encuestas), de sus prácticas anticonceptivas, del ejercicio de su sexualidad (aunque más cercano a la identificación de conductas de riesgo) ${ }^{12}$ y de las consecuencias negativas que tiene para los hombres ciertos modelos del ejercicio de la masculinidad. ${ }^{13}$ Como un objeto más de análisis derivado de estos debates, resaltan las temáticas diferenciadas entre los trabajos de los países del norte y los del sur; por ejemplo, en algunos casos en el norte se habla de la disminución en las diferencias de la esperanza de vida de las personas (en relación con una disminución en ciertas causas de muerte de los varones, algunas de las cuales son denominadas como patologías sociales), ${ }^{14}$ mientras que se reporta un mayor incremento en ciertas causas de mortalidad de las mujeres.

Ahora bien, estos hallazgos no necesariamente se interpretan a la luz de una crítica a los modelos de masculinidad dominantes en muchos contextos sociales, los cuales pueden estar asociados a lo que algunos autores han denominado una "negligencia suicida" o un "descuido intencional" de la propia salud por parte de los varones. Incluso en países como Canadá -donde los índices de desarrollo humano presentan niveles muy altos, colocando al país dentro de los primeros en el mundo de acuerdo a las clasificaciones de Naciones Unidas- se deja de lado el tema de la masculinidad..$^{15}$

En contraparte, si bien se presentó un trabajo sobre Nigeria relativo a las consecuencias negativas del ser hombre en términos de salud, hay otros trabajos sobre África (Kenia en particular) en donde simplemente se alude al consumo de alcohol como un factor de riesgo adicional de tener contactos sexuales no protegidos o, más grave aún, de contactos sexuales forzados, pero se problematiza poco sobre si dicho

${ }^{12}$ Susana Lerner, Olga Rojas y Mario Martínez, "Male fertility, reproductive valuations and sexual practices in Mexico: transformations under way?"; Normita Galban, "Male involvement in reproductive health concerns: the Philippine experience"; Juan Manuel Contreras, "El comportamiento reproductivo de los varones desde un enfoque sociodemográfico: algunos hallazgos a partir de la Encoplaf 96"; Cuong M. La, "Sexual behaviour and the value of partner virginity: a study of college-aged male Vietnamese students".

${ }^{13}$ Guillermo J. González-Pérez, María Guadalupe Vega-López, Armando Muñoz, Carlos E. Cabrera-Pivaral, Ana Valle Barbosa y Samuel Romero Valle, "El precio de la masculinidad: sobremortalidad masculina por homicidios en México"; Clifford O. Odimegwu, Christian Okemgbo y Saseendran Pallikadavath, "What is the cost of being a man? An analysis of social and health consequences of masculinity in Nigeria".

${ }^{14}$ Frank Trovato y N. M. Lalu, "A continuing pattern of decline of the sex differential in life expectancy in Canada: Early 1970s - Late 1990s”.

${ }^{15}$ Idem. 
consumo está asociado a modelos de masculinidad, como se ha documentado en mucha literatura. ${ }^{16}$

Un trabajo muy interesante presentado por investigadoras francesas muestra que cuando se considera de manera explícita el ejercicio de la masculinidad permeado en muchos lugares por la violencia, se evalúan temas como la mortalidad materna de una manera más sutil, definiendo nuevos indicadores para hacer visibles las problemáticas alrededor de un evento no deseado, ${ }^{17}$ y para el cual se han identificado diferentes formas de evitarlo mediante la definición de políticas públicas, servicios de salud y educativos, y censuras de diferentes características.

Ello complementa y a la vez diversifica las observaciones que algunos investigadores de la UNAM presentaron sobre el tema de la violencia durante el embarazo. ${ }^{18}$ Además muestra la necesidad de que los objetos de estudio de la demografía sean revisados y repensados constantemente a la luz de los avances temáticos, de los paradigmas vigentes, de las metodologías que se van desarrollando; todo ello con el propósito de que la investigación en este ámbito del conocimiento tenga de manera permanente las características de ser una reflexión ordenada, sistemática y crítica a través de la cual se genera conocimiento. ${ }^{19}$

Una de las modalidades del análisis de la población masculina en los procesos reproductivos presentada en la reunión se refiere a las licencias laborales que se otorgan alrededor de un parto en primera instancia y después durante el proceso de crianza y educación de los hijos. ${ }^{20}$ En la mayor parte de los países se da como obvio que dicha licencia es una prerrogativa de las madres trabajadoras para que pue-

${ }_{16}$ Tilda Farhat, Carolyn Tucker Halpern y Elle M.H. Mitchell, "Alcohol use, khat smoking and risky sexual behaviour among high school boys in Nairobi, Kenya: findings from a web-based survey".

${ }^{17}$ Myriam Khlat y Agnes Guillaume, "Evolution des concepts de mortalité maternelle et émergence des morts violentes en relation avec la grossesse"; Arlette Gautier, "Marriage laws and violence against women".

${ }^{18}$ Roberto Castro e Irene Casique, "Violencia de parejas contra mujeres en México. Un análisis comparativo entre varias encuestas nacionales".

${ }^{19}$ Laura Bernardi, Sylvia Keim y Holger Von der Lippe, "Improving the comparability of qualitative research designs to investigate social influence on fertility"; Grit Beck, "Interpretation of qualitative data: suggestions for moving back and forth between within-case and across-case analysis".

${ }^{20}$ Therese M. Jacobs, "Becoming a parent: material and immaterial consequences in the 90's"; Sara Brachet, "Le congé parental en Suède et ses conséquences sur le partage des charges parentales"; Germain Bingoly-Liworo y Evelyne Lapierre-Adamcyk, "Devenir parent au Canada: l'effet du prolongement des etudes"; Anna K. Meyer, "Does bargaining power influence the sharing of temporary parental leave?"; Nada Stropnik y Joze Sambt, "Parental leave and child benefit: attitudes, preferences and possible impact". 
dan recuperarse fisiológicamente del proceso del embarazo y del parto, y alimenten a su hijo o hija en sus primeras semanas de vida. Es menos frecuente que se cuestione la pertinencia de que los padres tengan una prestación similar debido, entre otras razones, a los roles diferenciados que tradicionalmente se han asignado para varones y mujeres alrededor de las actividades de la reproducción, y a la limitada definición de categorías, conceptos y marcos analíticos para interpretar las experiencias reproductivas de los varones. ${ }^{21}$

En ese sentido fue interesante escuchar la experiencia de investigadores, y sobre todo investigadoras, de países donde tanto las mujeres como los varones puedan optar por esa prestación, incluso por periodos de varias semanas después del parto (a diferencia de lo que sucede en otros donde sólo se otorgan dos o tres días, o una semana cuando mucho). Sin embargo, y a pesar de los avances en otros indicadores (como el de desarrollo humano y la equidad de género), no son tan altos los porcentajes de varones que recurren a la licencia por paternidad. ${ }^{22}$ Ello llevó a que en algunos debates se propusiera como política pública la obligatoriedad de que los hombres utilizaran tal prestación, visto incluso como una acción afirmativa para apoyar los derechos de las mujeres. Otra variante de esta reflexión es el de un análisis llevado a cabo en Polonia, donde se muestra que las políticas públicas parecieran estar orientadas a cierto tipo de modelo de familia más estereotipado, haciendo invisible con ello un espectro más amplio de formas de familia. Es evidente que para poder hacer este planteamiento es necesario aceptar una tipología que recupere la heterogeneidad de los arreglos familiares. ${ }^{23}$

Esta vertiente de reflexiones estuvo muy ligada a los debates sobre el uso del tiempo individual, familiar y de pareja, y la negociación del mismo en la cotidianidad; ello permeado por los roles de género y por los criterios del ejercicio del poder. ${ }^{24}$ Una variante más del cuestionamiento de ciertas especializaciones de género (consideradas por auto-

${ }^{21}$ Juan Guillermo Figueroa-Perea, "Les 'droits de l’être humain dans le domaine de la reproduction': une option pour repenser les comportements reproductifs des hommes dans le cadre des politiques démographiques".

${ }_{22}^{2}$ Anna K. Meyer, "Does bargaining power influence the sharing of temporary parental leave?"; Nada Stropnik y Joze Sambt, "Parental leave and child benefit: attitudes, preferences and possible impact".

${ }^{23}$ Anna Matysiak, "Sharing professional and household duties within the Polish couples-preferences and real choices".

${ }^{24}$ Evalyne Lapierre-Adamyck, Nicole Mareil Gratton y Celine Le Bourdais, "Temps parental et temps professional: la complamentarité des horaires de travail des parents". 
ras feministas como excluyentes y jerárquicas en detrimento de las mujeres) es el debate sobre el uso del apellido del esposo cuando las mujeres se casan (dentro de parejas heterosexuales), ${ }^{25}$ y las consecuencias que ello tiene en la asignación del apellido a los hijos e hijas productos de esa relación. Esto tiene matices distintos en los países donde se tiene la costumbre de utilizar un solo apellido; pero incluso en aquellos donde se utilizan dos, el hecho de privilegiar el del padre como primer apellido, acaba haciendo invisible la referencia a la madre de manera diferenciada para los hijos y las hijas cuando forman a su vez una pareja. Además, aúnense las respuestas que llegan a dar los varones al justificar su deseo de tener hijos varones: como una manera de "mantener el apellido". En ningún trabajo se llegó a proponer que eso se resolviera cambiando el apellido, pero sí se cuestionaron los criterios que se han asumido como obvios para nombrar a las mujeres una vez que se casan.

$\mathrm{Al}$ respecto, y aunado a un interés personal vinculado con el ámbito en el que trabajo, es interesante destacar que algunas de las mujeres académicas que han utilizado el de su pareja como primer apellido, cuando llegan a divorciarse optan por seguirlo utilizando para evitar confusiones en cuanto a la autoría e identidad de sus trabajos.

Si bien las licencias de paternidad fueron discutidas más en la lógica del beneficio para los hijos que de los derechos de los propios hombres, se utilizaron argumentos sobre los beneficios que los varones pueden obtener por el hecho de convivir constantemente con los hijos desde sus primeras etapas de vida, en la lógica de los aprendizajes, las formas de hablar, de escuchar y de negociar, además de la parte lúdica de ese intercambio.

Se discutieron los modelos de convivencia en las relaciones entre varones y mujeres, y se hizo referencia a lo que pasa después de que las parejas se separan, ya que hay diferencias importantes en los procesos de volver a establecer uniones y tener hijos. Se comentó que dichas diferencias tienen interpretaciones importantes a partir de las especializaciones de género, incluso en países como los europeos donde si bien hay una larga tradición de que hombres y mujeres vivan solos desde edades muy tempranas, en el momento en que se divorcian o enviudan es mucho mayor el porcentaje de mujeres que viven solas. ${ }^{26}$ Adicional-

${ }^{25}$ Marie-France Valetas, "La transmission du nom de famille".

${ }^{26}$ Martine J.C. Corijn y Edith Lodewijckx, "Civil status dynamic and household position in Flanders (Belgium)"; Joëlle Gaymu, Christiane Delbes, Adrien Binet y Aline Desesquelles, "Determinants of living arrangements among older people: a European comparison". 
mente se llegó a bromear (paradójicamente con una buena dosis de seriedad) con que muchos hombres reconocen que al divorciarse o enviudar buscan a alguien para que realice las labores del hogar o que esté en casa y se haga cargo de la misma, mientras que en contraparte las mujeres prefieren a alguien para poder estar fuera de casa. No en balde algunas de mis amigas feministas llegan a plantear que la viudez es un derecho de las mujeres, más que un evento crítico de su historia.

Otros temas discutidos tienen que ver con las tensiones entre la autonomía y el empoderamiento de las mujeres en el ámbito de la reproducción, con el involucramiento de los varones. Las estadísticas muestran ciertos desfases en las expectativas y prácticas de ambos. ${ }^{27} \mathrm{Se}$ discutieron también esas categorías ya que en ningún momento se habla del empoderamiento de los hombres en la reproducción, en parte porque se asume que ya están empoderados bajo el supuesto de que siempre han tomado decisiones, pero también porque se piensa en un poder que al incrementarse en las mujeres debiera disminuir en los varones -lo cual sería muy cuestionable desde la perspectiva de Michel Foucault- por lo que más bien haría falta un replanteamiento de lo que se interpreta por poder y negociación en la reproducción.

Se documentaron casos de relaciones sexuales no consensuadas tanto al inicio de las relaciones maritales como en el origen de dichas relaciones, así como casos de abuso sexual infantil. Ello pone nuevamente sobre la mesa de discusión la necesidad de analizar los comportamientos reproductivos en un entorno sexual permeado por relaciones de poder e identidades de género, y cuestionar profundamente las visiones asexuadas tanto de algunas aproximaciones académicas como de la definición de políticas públicas sobre el tema. ${ }^{28}$

Se presentaron trabajos sobre la historia de la fecundidad masculina, ${ }^{29}$ lo cual es una novedad pues es un tema poco documentado en múltiples espacios nacionales; también se aludió a la fragilidad de la masculinidad, a la "epidemia de accidentes de tránsito" en Brasil, ${ }^{30} \mathrm{a}$

${ }^{27}$ Britta C. Beenhakker y Michelle J. Hindin, "Women's autonomy and male involvement in antenatal care: associations and tensions"; Andrzej Kulczycki, "He said, she said: husband-wife agreement, power relations, and contraceptive use in Turkey".

${ }^{28}$ Ann M. Moore, Kofi Awusabo, Johannes John-Langba, Akwasi Kumi-Kyereme y Nyovani Madise, "Coercive first sex among adolescents in Sub-Saharan Africa"; Rajib Acharya, Michael Koening y Rabindra Kumar Sinha, "Prevalence and risk factors for intimate partner sexual violence: new evidence from rural India"; Cicely A. Marston, "Child sexual abuse in Mexico: a descriptive, qualitative study".

${ }^{29}$ Fabienne Daguet, "History of male fertility in France during the Xx century".

${ }^{30}$ Ana Maria Nogales Vasconcelos y David Duarte Lima, "Traffic accident mortality in Brazil; a silent tragedy". 
los prejuicios de los investigadores al no abordar ciertas temáticas como la infertilidad masculina, ${ }^{31}$ entre otros muchos elementos que han ido definiendo la agenda de investigación alrededor del comportamiento reproductivo y el lugar que en ella ocupa la referencia a los varones.

Por último, me gustaría resaltar de manera breve algunos temas que parecieran ser todavía una problemática aparentemente distante en los contextos latinoamericanos, en particular en el mexicano, pero que dados los procesos de globalización y de desarrollo en las diferentes sociedades, es probable que en un futuro cercano sean objeto de investigación también en México. Tal es el caso, por ejemplo, de análisis sofisticados que se presentan sobre la relación entre el uso de antidepresivos y el suicidio, o sobre los determinantes socioeconómicos del mismo, ${ }^{32}$ para lo cual no únicamente se requiere cierta incidencia del fenómeno, sino que haya una información disponible.

Otra variante es el caso de indicadores sobre tratamientos de reproducción asistida, tanto en función del número de nacimientos que en total se tiene, como en función de ciertos montos de la población nacional dentro de la cual se sigue esta práctica. ${ }^{33}$ Algunos estudios en Europa mostraban, por ejemplo, que la tendencia de muchas mujeres a posponer el inicio de la fecundidad tiene relación con eventos de infertilidad, por lo que existe interés por la reproducción asistida no sólo en el ámbito individual sino en el social. Incluso hay mujeres que recurren a la congelación de sus óvulos a fin de utilizarlos en el futuro, lo cual conlleva no sólo nuevas demandas para el avance tecnológico, sino también dilemas éticos. ${ }^{34}$

Como señalaba al principio, éste no pretende ser un recuento general del contenido de la Conferencia, sino una reflexión personal sobre algunos temas que me llamaron la atención a partir de mi propia experiencia de investigación.

${ }^{31}$ Sabine Gross, Walter Krause y Ulrich Mueller, "Life time mortality of men with normal and with subnormal sperm counts as young adults".

${ }^{32}$ Annette Erlangsen y Vladimir Cabudas-Romo, "Change in suicide rate attributable to individual's treatment with antidepressants"; Netta Mäki y Pekka Martikainen, "Socio-economic differences in suicide mortality by sex in Finland, 1971-2001".

${ }^{33}$ Pascale Donati, "Infécondité volontaire et involontaire"; Maria Santiago Delefosse, "Procréation Médicalement Assistée et vouloir mère"; Catherine Villeneuve-Gokalp, "Adoption et désir d' enfant".

${ }^{34}$ Pascale Donati, "Du choix de la non procréation aux rendez-vous manqués avec l'enfant"; Alessandro Rosina y Giulia Rivellini, "On the association between late parental age and the risk of stillbirth: evidence from northern Italy". 